\title{
Intravenous patient-controlled fentanyl with and without transversus abdominis plane block in cirrhotic patients post liver resection
}

This article was published in the following Dove Press journal:

Local and Regional Anesthesia

29 May 2014

Number of times this article has been viewed

\author{
Manar Serag Eldin' \\ Fatma Mahmoud' \\ Rabab El Hassan ${ }^{2}$ \\ Mohamed Abdel Raouf' \\ Mohamed H Afifi ${ }^{2}$ \\ Khaled Yassen' \\ Wesam Morad ${ }^{3}$ \\ 'Department of Anaesthesia, Liver \\ Institute, ${ }^{2}$ Department of Anaesthesia, \\ Faculty of Medicine, ${ }^{3}$ Department \\ of Community Medicine and Public \\ Health, Liver Institute, Menoufiya \\ University, Shebin El-Kom, Egypt
}

Background: Coagulation changes can complicate liver resection, particularly in patients with cirrhosis. The aim of this prospective hospital-based comparative study was to compare the postoperative analgesic efficacy of intravenous fentanyl patient-controlled analgesia (IVPCA) with and without transversus abdominis plane (TAP) block.

Methods: Fifty patients with Child's A cirrhosis undergoing liver resection were randomly divided into two groups for postoperative analgesia, ie, an IVPCA group receiving a $10 \mu \mathrm{g} / \mathrm{mL}$ fentanyl bolus of $15 \mu \mathrm{g}$ with a 10-minute lockout and a maximum hourly dose of $90 \mu \mathrm{g}$, and an IVPCA + TAP group that additionally received TAP block ( $15 \mathrm{~mL}$ of $0.375 \%$ bupivacaine) on both sides via a posterior approach with ultrasound guidance before skin incision. Postoperatively, bolus injections of bupivacaine $0.375 \%$ were given every 8 hours through a TAP catheter inserted by the surgeon in the open space during closure of the inverted L-shaped right subcostal with midline extension (subcostal approach) guided by the visual analog scale score $(<3,5 \mathrm{~mL}$; 3 to $<6,10 \mathrm{~mL} ; 6-10,15-20 \mathrm{~mL}$ ) according to weight (maximum $2 \mathrm{mg} / \mathrm{kg}$ ). The top-up dosage of local anesthetic could be omitted if the patient was not in pain. Coagulation was monitored using standard coagulation tests.

Results: Age, weight, and sex were comparable between the groups $(P>0.05)$. The visual analog scale score was significantly lower at $12,18,24,48$, and 72 hours $(P<0.01)$ in IVPCA + TAP group. The Ramsay sedation score was lower only after 72 hours in the IVPCA + TAP group when compared with the IVPCA group $(1.57 \pm 0.74$ versus $2.2 \pm 0.41$, respectively, $P<0.01)$. Heart rate, systolic blood pressure, and fentanyl consumption were lower in the IVPCA + TAP group at 24,48 , and 72 hours $(P<0.05)$. Intensive care unit stays were significantly shorter with TAP ( $2.61 \pm 0.74$ days versus $4.35 \pm 0.79$ days, $P<0.01)$. Prothrombin time and International Normalized Ratio indicated temporary hypocoagulability in both groups.

Conclusion: Combining TAP with IVPCA improved postoperative pain management and reduced fentanyl consumption, with a shorter stay in intensive care. TAP block can be included as part of a balanced multimodal postoperative pain regimen.

Keywords: transversus abdominis plane block, liver resection, postoperative pain

\section{Introduction}

Patients with cirrhosis are frequent candidates for liver-related surgery at our Liver Institute in Egypt, and providing optimal postoperative pain relief in these patients is a challenge for the anesthetic team. The use of neuraxial techniques and/or intravenous opioids has been a matter of debate for a number of years because of the wide range of modalities used for pain management at various research centers in different parts of the world.
Anaesthesia Department, Liver Institute, Menoufiya University, Gamal Abdel Nasser Street, Shebin El-Kom,

Menoufiya, Egypt

Tel +20106308 0170

Email kyassen6I@hotmail.com 
Postoperative pain is of major concern for patients undergoing upper abdominal surgery, in particular liver resection, which involves retraction of the ribs to facilitate surgical exposure. The potential development of post-liver resection coagulopathy has been reported in the past, so there is a tendency now to avoid epidural analgesia. Alternative methods are being developed to ensure a longer and safer postoperative analgesia period, including intravenous patientcontrolled opioid analgesia (IVPCA), and more recently, the transversus abdominis plane (TAP) block. However, few relevant studies have been performed in patients with cirrhosis undergoing liver resection. Pain management techniques need revision in order to achieve the advantageous effects of pain relief without unnecessary side effects.

IVPCA using opioids has become an accepted technique for pain control following major abdominal surgery, but the high doses of opioids required in this regimen are undesirable in patients with cirrhosis scheduled for liver resection and expected to have temporary liver dysfunction in the immediate postoperative period as a result of trauma to the liver parenchyma. ${ }^{1,2}$ TAP block is a method whereby the sensory afferents supplying the anterior abdominal wall are blocked using local anesthetic agents, and over the past few years has been gaining popularity as an alternative analgesic technique in patients undergoing major abdominal surgery..$^{3-6}$

The aim of this prospective hospital-based comparative study was to assess the postoperative analgesic efficacy of IVPCA with and without TAP in patients with Child's A cirrhosis undergoing liver resection, to identify any related side effects, and assess the impact of TAP block in a multimodal postoperative analgesic regimen.

\section{Patients and methods}

After receiving approval (0068/2013) from the local ethics committee of the Liver Institute at Menoufiya University in Egypt, as well as written informed consent, 50 patients with class A cirrhosis according to the Child-Pugh classification undergoing liver resection and requiring acute pain management during the immediate postoperative period were enrolled into this study and randomized using the sealed envelope technique to receive either IVPCA (fentanyl $10 \mu \mathrm{g} / \mathrm{mL}$ ) or IVPCA + TAP (fentanyl $10 \mu \mathrm{g} / \mathrm{mL}$ and bupivacaine $0.375 \%$ ).

All operations were performed under general anesthesia. The patients were well educated about the syringe pump for patient IVPCA and how to use it. A detailed description and explanation of the pain management plan was provided for each patient and enough time was given for them to inquire about their management and to provide their informed consent.

Inclusion criteria included provision of written informed consent, age 25 years or older, not on any existing anticoagulant or opioid therapy, being scheduled for elective liver resection surgery, Child A according to the Child-Pugh classification, and American Society of Anesthesiologists score 2 or 3. Exclusion criteria included objection to TAP block or inability to use a patient-controlled analgesia (PCA) machine, a serious intraoperative complication such as severe hemorrhage that could affect the level of consciousness and hence cooperation, need for postoperative ventilation, psychiatric disease, and termination of surgery due to inoperable status, for example, extension of tumor or pathology beyond the proposed resection limits. Patients who had developed opioid tolerance due to chronic use were also excluded. Data reported included age, sex, indication for surgery, intraoperative requirement for blood products, and length of stay in the intensive care unit. A complete blood count and prothrombin time were determined preoperatively and for 3 days postoperatively. Noninvasive and invasive hemodynamics were monitored perioperatively.

\section{IVPCA group}

On emergence from anesthesia, each patient was transferred to the intensive care unit and commenced on IVPCA. The PCA pump was programmed to deliver a $15 \mu \mathrm{g}$ bolus of fentanyl on demand, with a 10-minute lockout interval and a maximum hourly fentanyl dose of $90 \mu \mathrm{g}$. The background infusion was set to nil. All settings were saved and keylocked. Postoperative use of and requests for analgesia were recorded for later analysis.

\section{IVPCA + TAP group}

General anesthesia was induced in the same manner as for the IVPCA group, except that a single dose of TAP block was administered on both sides after preparing the skin with povidone iodine $10 \%$ solution and before skin incision. TAP block is a regional anesthetic technique that blocks sensation to the anterior abdominal wall. All blocks were performed by the same anesthesia consultant, who had received training in ultrasound-guided regional analgesia blocks at the inaugural James Cook Regional Anaesthesia Course in Egypt in 2010 organized by Durham University, UK.

A linear high-frequency transducer $(5-10 \mathrm{mHz})$ ultrasound probe (NanoMaxx ${ }^{\circledR}$; Sonosite Inc., Hitchin, UK) was used to guide the TAP block because the relevant anatomical structures are relatively shallow. Using this technique, 
the transducer is placed in an axial (transverse) plane above the iliac crest and in the region of the anterior axillary line, while the ultrasound probe is placed in a transverse plane at the lateral abdominal wall in the mid axillary line, between the lower costal margin and the iliac crest, to guide a posterior TAP block. ${ }^{7}$

The TAP block is an intermuscular block between the internal oblique and transversus abdominis muscles. The sensory fibers located in this plane are difficult to visualize on ultrasound. The terminal branches of the anterior rami of T7-L1 are expected to lie within the TAP between the internal oblique and the transverse abdominis muscles above the iliac crest. Of these three muscles, the internal oblique muscle usually has the most prominent layer. A 22 gauge spinal needle was introduced in the plane of the ultrasound beam and directed towards the TAP (posterior approach). On entering the plane, $15 \mathrm{~mL}$ of bupivacaine $0.375 \%$ (maximum $2 \mathrm{mg} / \mathrm{kg}$ ) was injected as a local anesthetic on both sides and could be observed as dark oval shapes. Before final closure of the abdomen, an 18 gauge epidural catheter was inserted in the TAP by the surgeon (open TAP with a subcostal approach) to be used during the first 3 postoperative days for administration of local anesthetic. The position of the catheter is equivalent anatomically to the subcostal approach, with a filter attached and the catheter taped to the chest wall.

Postoperatively, all patients were transferred to the intensive care unit and started on the IVPCA regimen. The IVPCA + TAP group additionally received bolus injections of bupivacaine $0.375 \%$ through the TAP catheter guided by the patient's visual analog scale (VAS) score $(<3,5 \mathrm{~mL}$; 3 to $<6,10 \mathrm{~mL} ; 6-10,15-20 \mathrm{~mL}$ according to weight every 8 hours, with a maximum dose of $2 \mathrm{mg} / \mathrm{kg}$ allowed within an 8 -hour interval). The top-up dose of local anesthetic could be omitted if the patient was not in pain.

General anesthesia was induced in both groups of patients using fentanyl 1-2 $\mu \mathrm{g} / \mathrm{kg}$, propofol $1-2 \mathrm{~mL} / \mathrm{kg}$, and rocuronium $0.6 \mathrm{~mL} / \mathrm{kg}$ as a muscle relaxant. Anesthesia was maintained with desflurane in a $50 \%$ oxygen-air mixture. The intravenous muscle relaxant was administered as appropriate under the guidance of a nerve stimulator. The depth of general anesthesia was monitored using the Entropy ${ }^{\mathrm{TM}}$ system (GE, Helsinki, Finland) which monitors processed electroencephalographic changes and helps to avoid patient awareness. Intraoperative monitoring included electrocardiography, pulse oximetry, capnography, analysis of fractional inspired $\mathrm{O}_{2}$, body temperature, urine output, central venous pressure, and invasive and noninvasive blood pressure measurements. Tidal volume and ventilation rate were adjusted to maintain an end tidal $\mathrm{CO}_{2}$ of 35-40 $\mathrm{mmHg}$. Intraoperative hypothermia was prevented by forced air-surface warming.

The surgical team was the same for all the procedures performed. A right subcostal surgical incision with extended supraumbilical extension (ie, inverted L-shape) was performed in all cases, and is considered to be less traumatic than the T-shaped incision. An ultrasonic dissector was used to divide the liver parenchyma. No Pringle maneuver was performed. The middle hepatic vein was preserved. Measures to reduce intraoperative bleeding included maintaining a low positive central venous pressure during the resection procedure and careful parenchyma transection using a Cavitron ultrasonic surgical aspirator, bipolar electrocautery, and an Harmonic $^{\circledR}$ scalpel (Ethicon Endo-Surgery, Inc. Johnson \& Johnson, Blue Ash, Cincinnati, OH, USA).

Low molecular weight heparin (enoxaparin) was given subcutaneously at a dose of $40 \mathrm{mg}$ once daily as prophylaxis for deep vein thrombosis in all patients from the second postoperative day until hospital discharge, but was regularly revised according to coagulation studies. Any blood products given were recorded. All patients were extubated in the operating room before being transferred to the intensive care unit.

Postoperative pain was assessed using a $10 \mathrm{~cm}$ VAS (0-10; 0 indicating no pain and 10 indicating worst pain during movement, eg, inspiration or cough). The VAS had a $10 \mathrm{~cm}$ line, and each patient was asked to make a vertical mark on the line to indicate the intensity of their pain. ${ }^{8}$ Side effects, such as pruritus and postoperative nausea and vomiting, were scored as follows: 0 , none; 1 , yes, does not require treatment; 2 , yes, requires and relieved by treatment; or 3 , yes, but not relieved by treatment. The Ramsay sedation scale assesses arousability on six levels, ${ }^{9}$ as follows: 1 , patient is anxious and agitated, restless, or both; 2 , patient is cooperative, oriented, and tranquil; 3, patient responds to commands only; 4, patient shows a brisk response to light or loud auditory stimulus; 5 , patient shows a sluggish response to loud auditory stimuli; and 6, patient exhibits no response. Immediate cessation of IVPCA and administration of intravenous naloxone $40 \mu \mathrm{g}$ was required in the event of respiratory depression, defined as a respiratory rate of less than 10 breaths per minute. Postoperative pain was assessed using the VAS at $6,8,12$, and 24 hours, and again on days 2 and 3 postoperatively. Opioid consumption and length of stay in the intensive care unit were recorded for both groups of patients. The primary outcome was a comparison of the two anesthetic regimens with regard to pain control. Secondary outcomes included opioid consumption and complications, such as sedation, nausea, vomiting, pruritus, respiratory 
depression, and signs of local toxicity, as well as length of stay in the intensive care unit.

\section{Statistical analysis}

Twenty-five patients were allocated to each treatment group. Recruitment was based on the following assumptions: a power of $90 \%, \alpha=0.05$, and a ratio of cases to controls of $1: 1$. The required sample size was determined using power and sample size calculation software. The data were analyzed using Statistical Package for the Social Sciences for Windows version 13 software (SPSS Inc., Chicago, IL, USA). The data are shown as the mean and range or value and frequency with percentage. No intention to treat analysis was performed. Randomization was achieved using a closed envelope technique as mentioned earlier.

The Student's $t$-test was used to compare the mean and standard deviation for normally distributed quantitative variables, and a $P$-value $<0.05$ was considered to be statistically significant. The Mann-Whitney $U$ test was used for quantitative variables which were not normally distributed, and a $P$-value $<0.05$ was considered significant. The paired $t$-test was used to detect the mean and standard deviation of normally distributed pre and post values of the same variable in the same group of patients, and a $P$-value $<0.05$ was considered significant. The Wilcoxon test was used to detect the mean and standard deviation of non-normally distributed pre and post values of the same variable in the same group of patients, and a $P$-value $<0.05$ was considered significant. Repeated-measures analysis of variance was performed to differentiate changes in different follow-up results for normally distributed study variables, and a $P$-value $<0.05$ was considered significant. The Friedman test was performed to differentiate changes in different follow-up results for different study variables, and a $P$-value $<0.05$ was considered significant. All data were tested with the Kolmogorov-Smirnov Z test, and most were found to be normally distributed, and are therefore shown as the mean \pm standard deviation. Parametric tests were used when doing associations and correlations.

\section{Results}

Fifty-nine patients were enrolled, with nine patients excluded and replaced, two due to extension of the tumor beyond the resection limits with termination of the procedure (one patient required more than 6 units of packed red blood cells and similar units of plasma), four patients in the IVPCA + TAP group because of suspicion of intravascular migration of the TAP catheter, two due to the catheter falling out during postoperative wound dressing, and one because of a technical failure involving their PCA syringe pump.

Patient characteristics were comparable between the two groups. There were 15 males $(60 \%)$ and ten females $(40 \%)$ in the IVPCA group and 16 males (64\%) and nine females $(36 \%)$ in the IVPCA + TAP group $(P>0.05)$. There were no significant differences between the IVPCA and the IVPCA + TAP groups with regard to mean age and weight $(50.4 \pm 10.3$ years versus $49.24 \pm 14$ years and $81.6 \pm 15.7 \mathrm{~kg}$ versus $82.7 \pm 8.68 \mathrm{~kg}$, respectively, $P>0.05$ ). The frequency of surgery in the IVPCA group and IVPCA + TAP block group was six (24\%) and 17 (68\%), respectively, for major liver resection, and $19(76 \%)$ and eight (32\%) for minor liver resection. Mean surgical times were comparable between the groups, being $302 \pm 54$ minutes in the IVPCA group and $286 \pm 44$ minutes in the IVPCA + TAP group $(P>0.05)$. Ultrasound-guided preincision bilateral TAP blocks were not time-consuming and took a median of 19 (range 15-24) minutes for an experienced anesthetist to perform. Inserting and fixing a catheter in the open TAP space during closure of the abdominal muscles toward the end of surgery was also not time-consuming.

\section{Hemodynamics}

Some patients in each group required a transfusion of packed red blood cells, ie, three in the IVPCA group and four in the IVPCA + TAP group ( 2 units). Mean blood loss was $578.2 \pm 79.72 \mathrm{~mL}$ in the IVPCA group and $583.2 \pm 77.28 \mathrm{~mL}$ in the IVPCA + PCA group, with no significant difference between the groups $(P>0.05)$. As mentioned earlier, one patient was excluded due to tumor extension beyond the surgical resection limits and required more than 6 units of packed red blood cells during surgical mobilization of the liver. This patient was extubated in the intensive care unit after brief ventilatory support to control developing acidosis.

The 50 study patients demonstrated a significant improvement in hemodynamics postoperatively, indicating recovery from surgical stress on IVPCA during the 72-hour follow-up period. All vital signs normalized by the third postoperative day. In the IVPCA + TAP group, there was also a significant decrease in hemodynamics during the 72 hours of follow-up, with all vital signs normalizing by the third day. Comparison of the two groups with regard to heart rate and systolic/diastolic blood pressure showed significant differences in favor of the IVPCA + TAP group $(P<0.05$, Table 1$)$. 
Table I Hemodynamic differences between IVPCA and IVPCA + TAP groups

\begin{tabular}{|c|c|c|c|c|}
\hline $\begin{array}{l}\text { Study } \\
\text { variable }\end{array}$ & Groups & Mean \pm SD & $t$-test & $P$-value \\
\hline \multirow[t]{2}{*}{ HR POD I } & IVPCA & $94.29 \pm 13.4$ & 3.98 & $<0.01$ \\
\hline & IVPCA + TAP & $79.52 \pm 10.6$ & & \\
\hline \multirow[t]{2}{*}{ SBP POD I } & IVPCA & $121.7 \pm 13.12$ & 3.68 & $<0.01$ \\
\hline & IVPCA + TAP & $108.4 \pm 10.3$ & & \\
\hline \multirow[t]{2}{*}{ DBP POD I } & IVPCA & $77 \pm 8.69$ & 2.76 & $<0.01$ \\
\hline & IVPCA + TAP & $70 \pm 7.64$ & & \\
\hline \multirow[t]{2}{*}{ HR POD 2} & IVPCA & $94.35 \pm 15.3$ & 2.59 & $<0.01$ \\
\hline & IVPCA + TAP & $82.2 \pm 14.4$ & & \\
\hline \multirow[t]{2}{*}{ SBP POD 2} & IVPCA & $127.06 \pm 13$ & 5.08 & $<0.01$ \\
\hline & IVPCA + TAP & $106.25 \pm 12.8$ & & \\
\hline \multirow[t]{2}{*}{ DBP POD 2} & IVPCA & $74.53 \pm 9.35$ & 2.64 & $<0.01$ \\
\hline & IVPCA + TAP & $67.08 \pm 8.59$ & & \\
\hline \multirow[t]{2}{*}{ HR POD 3} & IVPCA & $93.69 \pm 14.5$ & 2.59 & $<0.05$ \\
\hline & IVPCA + TAP & $83.96 \pm 9.27$ & & \\
\hline \multirow[t]{2}{*}{ SBP POD 3} & IVPCA & $124.12 \pm 15.74$ & & \\
\hline & IVPCA + TAP & $109.38 \pm 12.19$ & 3.38 & $<0.01$ \\
\hline \multirow[t]{2}{*}{ DBP POD 3} & IVPCA & $73.24 \pm 6.6$ & & \\
\hline & IVPCA + TAP & $71.25 \pm 7.98$ & 0.84 & $>0.05$ \\
\hline
\end{tabular}

Notes: Data are presented as the mean \pm SD. The Student's $t$-test was used to compare HR, SBP, and DBP. $P<0.05$ was considered to be statistically significant and $P<0.0$ I was considered to be highly statistically significant.

Abbreviations: DBP, diastolic blood pressure; HR, heart rate; IVPCA, intravenous patient-controlled analgesia; SBP, systolic blood pressure; SD, standard deviation; $\mathrm{POD}$, postoperative day; TAP, transversus abdominis plane.

\section{Sedation}

The mean sedation score was significantly different between the IVPCA + TAP group $(1.57 \pm 0.47)$ and the IVPCA group $(2.2 \pm 0.41)$ on the third postoperative day $(P<0.01$, Table 2).

\section{Pain control}

The mean intraoperative dose of fentanyl administered (as guided by processed electroencephalography) was comparable between the IVPCA and IVPCA + TAP groups (370 $\pm 100 \mu \mathrm{g}$ versus $358 \pm 104.70 \mu \mathrm{g}$, respectively, $P=0.676$ ). Postoperatively, both groups showed similarly effective pain control at rest (VAS $<3$ ) using a multimodal analgesia regimen of IVPCA \pm TAP block and additional meperidine when requested. However, patients in the IVPCA + TAP group had more effective pain control at rest when coughing. The mean VAS score in the IVPCA + TAP group when coughing was $5 / 10$ on day $1,3 / 10$ on day 2 , and $3 / 10$ on day 3 , and in the IVPCA group was $7 / 10$ on day $1,6 / 10$ on day 2 , and $6 / 10$ on day $3(P<0.05$, Table 3$)$. Patients in both groups suffered breakthrough pain on coughing, particularly the IVPCA group, and were treated immediately with additional intravenous meperidine until pain was controlled.
Table 2 Postoperative sedation scores during follow-up in the IVPCA and IVPCA + TAP groups

\begin{tabular}{lllll}
\hline Time & Groups & $\begin{array}{l}\text { Median } \\
(\text { IQR) }\end{array}$ & $\begin{array}{l}\text { Mann- } \\
\text { Whitney } \\
\text { U test }\end{array}$ & P-value \\
\hline POD I & IVPCA & $4(3-4)$ & 0.25 & $>0.05$ \\
& IVPCA + TAP & $3(I-5)$ & & $>0.05$ \\
POD 2 & IVPCA & $2(I-2)$ & 0.21 & \\
& IVPCA + TAP & $2(I-2)$ & & $<0.01$ \\
POD 3 & IVPCA & $2(2-2)$ & 3.09 & \\
& IVPCA + TAP & I (I-2) & & \\
\hline
\end{tabular}

Notes: Data are presented as the median and IQR. The Mann-Whitney $U$ test was used to compare postoperative sedation scores. $P<0.01$ was considered to be highly statistically significant.

Abbreviations: IVPCA, intravenous patient-controlled analgesia; POD, postoperative day; TAP, transversus abdominis plane; IQR, interquartile range (25th percentile to 75th percentile).

\section{Additional drugs needed for pain}

No group was immune from the need for rescue intravenous drugs for breakthrough pain despite this occurring less frequently in the IVPCA + TAP group. Eight cases in the IVPCA group and six cases in the IVPCA + TAP group required additional rescue opioids in the form of intravenous boluses of meperidine $50 \mathrm{mg}$ for breakthrough pain on exertion, mainly when coughing (a total of $950 \mathrm{mg}$ over 3 days in the IVPCA group versus $400 \mathrm{mg}$ in the IVPCA + TAP group).

\section{Nausea and vomiting}

Three patients in the IVPCA group and one in the IVPCA + TAP group suffered from nausea and vomiting, which was

Table 3 Differences in postoperative pain scores between the IVPCA and IVPCA + TAP groups

\begin{tabular}{lllll}
\hline Time & Groups & $\begin{array}{l}\text { Median } \\
(\text { IQR) }\end{array}$ & $\begin{array}{l}\text { Mann- } \\
\text { Whitney } \\
\text { U test }\end{array}$ & \\
& & \multicolumn{1}{l}{ P-value } \\
\hline 6 hours on POD I & IVPCA & $3(3-4)$ & 3.5 I & $<0.0$ I \\
& IVPCA + TAP & $5(4-7)$ & & \\
I2 hours on POD I & IVPCA & $6(6-7)$ & 3.02 & $<0.0$ I \\
& IVPCA + TAP & $5(2-6)$ & & \\
I8 hours on POD I & IVPCA & $7(6-7)$ & 2.76 & $<0.0$ I \\
& IVPCA + TAP & $5(3-7)$ & & \\
24 hours on POD I & IVPCA & $7(6-7)$ & 4.25 & $<0.0$ I \\
& IVPCA + TAP & $5(4-5)$ & & \\
POD 2 & IVPCA & $7(6-7)$ & 4.45 & $<0.0$ I \\
& IVPCA + TAP & $3(2-4)$ & & \\
POD 3 & IVPCA & $6(5-7)$ & 4.5 I & $<0.0$ I \\
& IVPCA + TAP & $3(2-5)$ & & \\
\hline
\end{tabular}

Notes: Data are presented as the median and IQR (25th percentile to 75th percentile). The Mann-Whitney $U$ test was used for comparisons. $P<0.01$ indicates a highly statistically significant difference.

Abbreviations: IVPCA, intravenous patient-controlled analgesia; POD, postoperative day; TAP, transversus abdominis plane; IQR, interquartile range (25th percentile to 75th percentile). 
treated with intravenous metoclopramide $10 \mathrm{mg}$ given once on the first postoperative day. No signs of respiratory depression were reported in either group, and no signs of overdose or toxicity were reported with fentanyl or bupivacaine. No hypotensive episodes were encountered post injection of local anesthesia.

\section{Laboratory parameters}

Tables 4 and 5 indicate a significant increase in the International Normalized Ratio (INR) for prothrombin time and prothrombin concentration, with a significant decrease in hemoglobin, hematocrit, and platelets $(P<0.01)$.

\section{Opioid and local anesthetic consumption}

Local anesthetic consumption was significant reduced during the first 3 postoperative days. There was a significantly increased consumption of fentanyl in the IVPCA group when compared with the IVPCA + TAP group (Table 6 and Figure 1). The mean postoperative amounts of local anesthesia (bupivacaine $0.375 \%$ ) injected through the catheter placed by the surgeon in the open TAP were as follows: $94.7 \pm 25.33 \mathrm{mg}$ on postoperative day $1,83.4 \pm 20.78 \mathrm{mg}$ on postoperative day 2 , and $77.8 \pm 18.32 \mathrm{mg}$ on postoperative day 3 . Repeatedmeasures analysis of variance showed a significant decrease in local anesthetic consumption as levels of surgically induced stress decreased $(P<0.05)$.

\section{Length of stay in intensive care unit}

There was a significant decrease in the length of stay in the intensive care unit in the IVPCA + TAP group (Figure 2).

\section{Satisfaction score}

There was no significant difference between the two groups with regard to satisfaction with pain control. A considerable number of patients in both groups rated their satisfaction with pain control as good to fair (Table 7).

\section{Discussion}

The IVPCA + TAP group showed a significant decrease in VAS scores for pain during the immediate postoperative period, probably due to the additive effects of the local anesthetic drug infused in the TAP. This result is similar to that reported by other investigators. Sharma et $\mathrm{al}^{10}$ found that they were able to provide highly effective analgesia for up to 24 hours postoperatively using this technique, with no complications due to TAP block and prolongation of the time to first request for tramadol PCA in patients undergoing major surgical procedures involving a mid line abdominal wall incision. McDonnell et $\mathrm{al}^{11}$ and Elkassabany et al ${ }^{12}$ came to the same conclusion, ie, that addition of TAP block helped to provide effective pain relief.

Use of a catheter in the TAP group to enable repeated bolus injections, as in our study, was also documented by Allcock et al, who concluded that a continuous TAP block provides excellent analgesia and augments postoperative pain relief following major abdominal surgery, particularly when neuraxial analgesia is inadvisable. ${ }^{13}$

Recent data suggest a need to monitor patients at risk of liver impairment and to consider reducing the dose of analgesia, so continuous infusion was not implemented in our study; instead, we used 8-hourly repeat boluses into the catheter titrated to the VAS and tailored to each patient. The 8 -hour dose can be omitted if the patient is not in pain in order to reduce the potential risk of toxicity from overdose, given that these patients can develop transient liver dysfunction post liver resection. The guidance from the American Society of Regional Anesthesia is that a regional technique should be "tailored to the minimum mass of local anesthetic molecules necessary to achieve the desired clinical effect". Local anesthetic toxicity following TAP block had been reported in several studies. Griffiths et $\mathrm{al}^{16}$ recently reported a study showing that plasma concentrations of ropivacaine after TAP block can reach potentially neurotoxic

Table 4 Conventional blood tests for the IVPCA group

\begin{tabular}{|c|c|c|c|c|c|c|}
\hline Study variables & Preoperative & POD I & POD 2 & POD 3 & $\begin{array}{l}\text { Repeated- } \\
\text { measures } \\
\text { ANOVA }\end{array}$ & $P$-value \\
\hline INR & $1.18 \pm 0.1$ & $1.19 \pm 0.1$ & $1.3 \pm 0.1 * *$ & $1.4 \pm 0.1 * *$ & I,38I.6I & $<0.01$ \\
\hline Platelets & $201 \pm 90.21$ & $195.6 \pm 99.4$ & $182.7 \pm 88.2$ & $171.5 \pm 82.5^{* *}$ & 8.15 & $<0.05$ \\
\hline PC (\%) & $82.06 \pm 12.19$ & $80.68 \pm 12.3^{*}$ & $67.9 \pm 11.0 * *$ & $63.9 \pm 13.6 * *$ & 764.21 & $<0.01$ \\
\hline Hemoglobin (g/dL) & $12.18 \pm 1.16$ & $12.7 \pm 1.5$ & $1 \mathrm{I} \pm 1.2^{* *}$ & $11.8 \pm 1.4$ & $2,785.32$ & $<0.01$ \\
\hline Hematocrit (\%) & $36.18 \pm 3.24$ & $38.4 \pm 5 *$ & $36.7 \pm 5.8$ & $35.7 \pm 4$ & $2,235.01$ & $<0.01$ \\
\hline
\end{tabular}

Notes: The data are presented as the mean and standard deviation. Repeated-measures ANOVA was used for INR, PC, hemoglobin, and hematocrit, and the Friedman test was used for platelets. $P<0.05$ was considered to be statistically significant and $P<0.0$ I was considered to be highly statistically significant. *Significant with Ist reading (Preop); **Highly significant with Ist reading (Preop).

Abbreviations: ANOVA, analysis of variance; INR, International Normalized Ratio; PC, prothrombin concentration; POD, postoperative day; IVPCA, intravenous patientcontrolled analgesia; Preop, preoperative. 
Table 5 Conventional blood tests for the IVPCA + TAP group

\begin{tabular}{|c|c|c|c|c|c|c|}
\hline Study variables & Preoperative & POD I & POD 2 & POD 3 & $\begin{array}{l}\text { Repeated- } \\
\text { measures } \\
\text { ANOVA }\end{array}$ & $P$-value \\
\hline INR & $1.07 \pm 0.22$ & $1.2 \pm 0.19 *$ & $1.49 \pm 0.26 * *$ & $1.54 \pm 0.25 * *$ & $\mathrm{I}, 849.44$ & $<0.01$ \\
\hline Platelets & $205.24 \pm 84.2$ & $193.11 \pm 88.9$ & $187 \pm 106$ & $149.59 \pm 62 *$ & 21.56 & $<0.01$ \\
\hline PC (\%) & $90.41 \pm 14.9$ & $70 \pm 18.16 * *$ & $49.5 \pm 12.6 * *$ & $47.1 \pm 13.4^{* *}$ & 736 & $<0.01$ \\
\hline Hemoglobin ( $(g / d L)$ & $13.29 \pm 1.57$ & $12.14 \pm 1.7^{* *}$ & $12.25 \pm 1.8^{*}$ & $11.14 \pm 1.7^{* *}$ & I,3II.95 & $<0.01$ \\
\hline Hematocrit (\%) & $37.83 \pm 4.39$ & $36.69 \pm 5.09$ & $37.15 \pm 55$ & $34.1 I \pm 5.4$ & I,396.22 & $<0.01$ \\
\hline
\end{tabular}

Notes: The data are presented as the mean and standard deviation. Repeated-measures ANOVA was used for INR, PC, hemoglobin, and hematocrit, and the Friedman test was used for platelets. $P<0.05$ was considered to be statistically significant and $P<0.01$ was considered to be highly statistically significant. *Significant with Ist reading (Preop); **Highly significant with Ist reading (Preop).

Abbreviations: ANOVA, analysis of variance; INR, International Normalized Ratio; PC, prothrombin concentration; POD, postoperative day; IVPCA, intravenous patientcontrolled analgesia; TAP, transversus abdominis plane block; Preop, preoperative.

levels in plasma. Nash et $\mathrm{al}^{14}$ demonstrated a reduction in postoperative opioid requirement and/or pain scores following TAP block with bupivacaine, and reported this with the injection of significantly less local anesthetic than they had intended when designing their study. In our study, local anesthetic drug boluses were only given when no fresh blood was being withdrawn from the TAP catheter, which could explain the low incidence of signs of toxicity. The surgical wound and spaces are vascular and so they have great potential to absorb significant amounts of the local anesthetic reagents. ${ }^{14-18}$

The decrease in opioid requirements in the IVPCA + TAP group during the early postoperative period reflects the opioids' sparing effect on the TAP block, which should be of particular benefit in patients with cirrhosis to avoid unnecessary doses of opioids post resection when temporary liver dysfunction is known to occur. In a meta-analysis of the clinical effectiveness of TAP block, Siddiqui et al $^{19}$ came to the same conclusion, ie, that TAP block reduces the requirement for postoperative opioids and is comparable with morphine in terms of postoperative analgesia. They concluded that TAP block increased the time to first request for further analgesia,

Table 6 Differences in fentanyl consumption in micrograms between the IVPCA and IVPCA + TAP groups

\begin{tabular}{llllll}
\hline $\begin{array}{l}\text { Fentanyl } \\
\text { consumption }\end{array}$ & Group & Mean & SD & t-test & P-value \\
\hline POD I & IVPCA + TAP & $1,000.80$ & 61.57 & 3.6 & $<0.01$ \\
& IVPCA & $1,254.6$ & 287.15 & & \\
POD 2 & IVPCA + TAP & 995.80 & 65.92 & 2.75 & $<0.05$ \\
& IVPCA & $1,293.29$ & 445.10 & & \\
POD 3 & IVPCA + TAP & 956.80 & 102.23 & 2.46 & $<0.05$ \\
& IVPCA & $1,182.24$ & 372.41 & & \\
\hline
\end{tabular}

Notes: The data are presented as the mean and standard deviation. The Student's $t$-test was used to compare fentanyl consumption in the two groups. $P<0.05$ was considered to be statistically significant and $P<0.01$ was considered to be highly statistically significant.

Abbreviations: IVPCA, intravenous patient-controlled analgesia; POD, postoperative day; TAP, transversus abdominis plane; SD, standard deviation. provided more effective pain relief, and reduced the risk of opioid-associated side effects. ${ }^{19}$

Brady et al studied the effect of open TAP block following right hemicolectomy, reporting that open TAP blocks are safe and reduce postoperative opioid requirements and sedation in patients undergoing this procedure. ${ }^{20}$ This decrease in opioid requirements with TAP block was echoed in a study by Milan et al, who used the subcostal approach for TAP block in liver transplant recipients and reported that the initial evaluation of subcostal TAP block after orthotopic liver transplant indicated a significant reduction in postoperative morphine consumption. ${ }^{21}$

The primary goal of our current study was to control postoperative pain, and this was successfully achieved by TAP block, both at rest and during coughing. Fentanyl consumption was only reduced by $20 \%$, but the decrease was statistically significant. TAP block was able to be included as part of a multimodal regimen, and our results should encourage further studies involving other peripheral nerve blocks, such as combined subrectal sheath and TAP in an attempt to block crossover of sensory fibers from the other side of the abdomen wall. This study is a step in our learning curve towards understanding and achieving better pain control for patients with cirrhosis undergoing liver resection. In the future we plan to study patients with more advanced liver disease, particularly those with strangulated hernia as a result of tense ascites undergoing emergency surgery, who always present an anesthetic challenge.

As already mentioned, bilateral TAP block was not timeconsuming when done by an experienced anesthetist, and took approximately 20 minutes to complete. Inserting a catheter into the open TAP space during closure of the abdominal muscles towards the end of surgery and fixing it was also not time-consuming, being essentially no different from fixing a biliary stent from the surgeon's point of view. In contrast with our findings, Sandeman et al reported that TAP block increased the anesthesia 
A

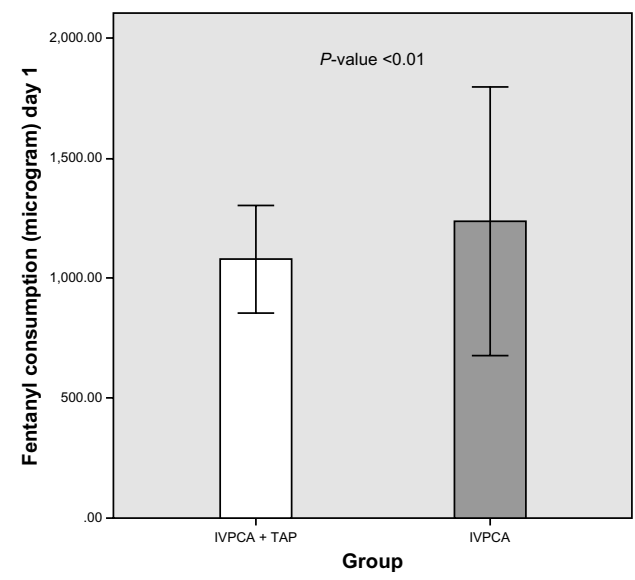

B

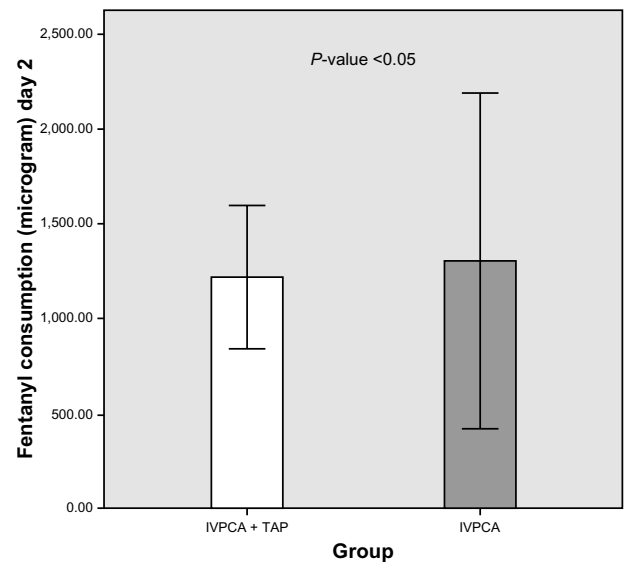

C

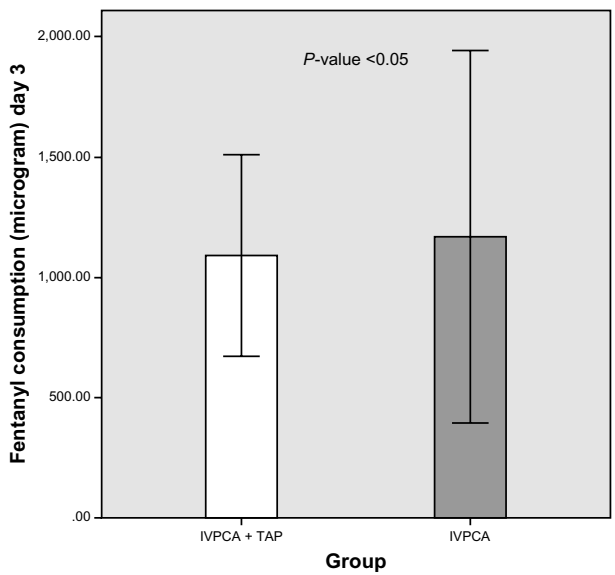

Figure I Bar chart showing the mean and standard deviation values for fentanyl consumption in the IVPCA and IVPCA + TAP groups. P $<0.05$ indicates statistical significance. A, $\mathrm{B}$, and $\mathrm{C}$ show fentanyl consumption on day $\mathrm{I}, 2$, and 3 , respectively.

Abbreviations: IVPCA, intravenous patient-controlled analgesia; TAP, transversus abdominis plane.

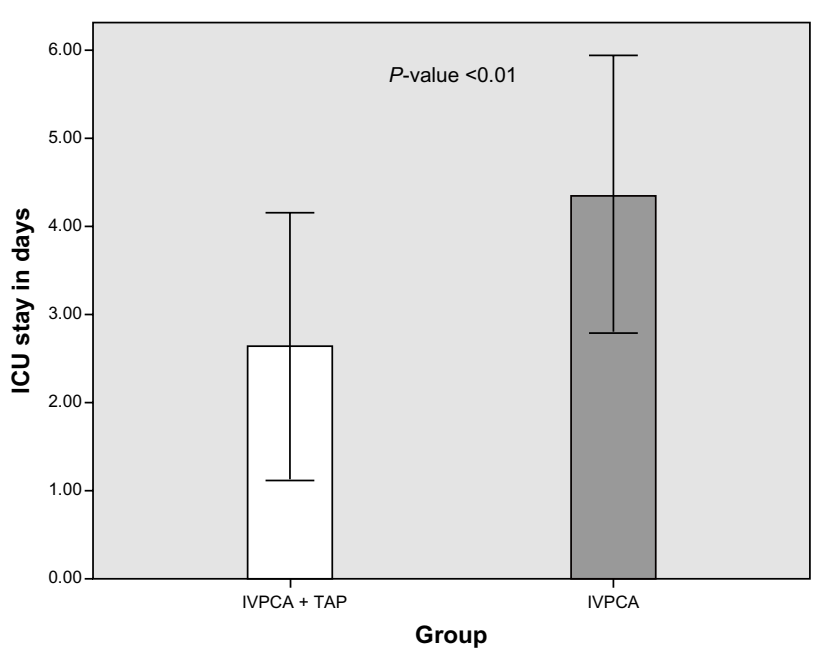

Figure 2 Bar chart showing the mean and standard deviation values for ICU stay between the IVPCA and IVPCA + TAP groups. $P<0.05$ indicates statistical significance.

Abbreviations: IVPCA, intravenous patient-controlled analgesia; TAP, transversus abdominis plane; ICU, intensive care unit. time by 14 minutes on average and offered no clinically important benefit over infiltration of local anesthetic at the port site in pediatric patients undergoing laparoscopic appendectomy, but this may be explained by the age of the cases (range 7-16 years) and the decreased pain threshold in this age group..$^{22}$ Another study by Freir et al also contradicted the previously mentioned studies, demonstrating no additional benefit of TAP block post renal transplant and no reduction in morphine requirements. ${ }^{23}$

In our study, the TAP catheter was inserted at the completion of surgery by the surgeon under supervision in the transversus abdominis muscle plane. This technique was also attempted by Zhong et al, who reported that intermittent delivery of bupivacaine through the TAP block catheter significantly reduced postoperative parenteral opioid requirements following free flap reconstruction of the breast. ${ }^{24}$

Multimodal analgesia can improve pain control and possibly improve the quality of recovery. De Oliveira et al reported 
Table 7 Differences in satisfaction scores between the IVPCA and IVPCA + TAP groups

\begin{tabular}{lllll}
\hline $\begin{array}{l}\text { Satisfaction } \\
\text { score }\end{array}$ & $\begin{array}{l}\text { IVPCA } \\
\text { TAP }\end{array}$ & IVPCA & $\chi^{2}$ test & P-value \\
\hline Excellent & $5(20.0)$ & $4(16.0)$ & 0.36 & $>0.05$ \\
Good & $9(36.0)$ & $11(44.0)$ & & \\
Fair & II (44.0) & $10(40.0)$ & & \\
Poor & $0(0.0)$ & $0(0.0)$ & & \\
\hline
\end{tabular}

Notes: The data are presented as the number (\%). $P$-value $>0.05$ using the $\chi^{2}$ test. Abbreviations: IVPCA, intravenous patient-controlled analgesia; TAP, transversus abdominis plane.

that TAP block is an effective adjunct in a multimodal analgesic strategy for ambulatory laparoscopic procedures. ${ }^{25}$ The findings of our current study demonstrate the beneficial effect of the TAP block as a contributor in a multimodal pain regime when combined with intravenous opioids for postoperative pain relief. However, the TAP block cannot be used as a sole method to control pain as it only affects the somatic sensory nerves and not the visceral. Unfortunately, this was reflected in our study by the considerable percentage of fair scores with regard to patient satisfaction. Improved technique and use of a subcostal rather than posterior approach preoperatively for pre-emptive analgesia could produce better results. A further development of our technique and learning curve would be to combine an open subrectal sheath block with open TAP in an attempt to block sensory fibers from the other side, given that one of the limitations of this study was the one-sided TAP block. The use of minimally invasive peripheral anterior abdominal wall nerve blocks is expected to be the focus of future studies dedicated to improving postoperative pain management regimes at the expense of the other traditionally used central neuroaxial blocks such as epidural analgesia.

Another limitation of our current study is the inability to alter the settings on the PCA pump in both groups during the 3 days of the study to meet with the demands for pain relief, so intravenous rescue opioids were used instead. Patients in both groups reported an increase in intravenous opioid consumption on day 2 due to the increased frequency of movement and physiotherapy, with no alteration in the pump settings. Further studies are needed to determine the optimal concentrations and settings of the PCA pump for this group of patients. The need for analgesia decreased in both groups on day 3 , and this could be attributed to the decrease in postoperative surgical stress response.

Our current study showed the need to use additional intravenous analgesics beside both protocols of patient controlled intravenous fentanyl with and without TAP block. The efficacy of each technique could be increased by using another adjuvant method of pain relief and help to maximize the efficacy of pain control.

Several other groups have reported effective pain control when a multimodal approach to postoperative pain control is adopted; for example, the study by Ozalp et al, who used different mixtures of local anesthetic drugs and opioids for better pain control. ${ }^{26}$ The amount of local anesthetic drug consumed varied from patient to patient in our study, where we titrated the dose according to VAS pain score to avoid giving excessive doses of local anesthesia. This could explain why there was no local anesthetic toxicity in our study. As mentioned earlier, the American Society of Regional Anesthesia advises that a regional technique should be "tailored to the minimum mass of local anesthetic molecules necessary to achieve the desired clinical effect". This is necessary from our point of view to prevent local anesthetic toxicity following TAP block, which has been reported and discussed in several other recent pulbications. ${ }^{14-17}$ Most of the studies reporting development of local toxicity post TAP used large volumes of local anesthesia. The TAP is a vascular space between the anterior abdominal muscles and is known to have the potential to absorb significant amounts of locally injected anesthetic agents, particularly when top-up doses are given at fixed time intervals. None of these studies included patients with cirrhosis undergoing major liver resection like those in our study. Future research is needed to investigate the actual plasma levels of local anesthetic injected when TAP block is performed in this patient population.

Use of a well designed wound infiltration catheter with multiple openings distributed over a longer segment of the catheter, instead of the widely available epidural set used in our study, which has fewer openings for perfusion, could improve the spread of the local anesthetic agent in the TAP space.

Most of the patients in our study were started on oral sips of water and juice on the first postoperative day, with a low incidence of nausea and vomiting in both the IVPCA group (3/17) and the IVPCA + TAP group (7/28); fewer patients overall needed medical intervention for symptomatic relief, and this may be attributed to the choice of fentanyl as the anesthetic agent, which is known to have a lower incidence of postoperative nausea and vomiting than morphine. This finding was in agreement with Hutchinson et $\mathrm{al}^{27}$ who found that IVPCA fentanyl had a significantly lower rate of common opioid side effects (nausea/vomiting) when compared with other methods of analgesia.

We found a decrease in sedation score during the first 3 postoperative days in both groups, with the highest scores 
recorded on the first day, and this could be attributed to the residual effect of the agents used for general anesthesia. Improvement in sedation scores was associated with a decrease in severity of pain on the subsequent postoperative days and a decrease in drug consumption. Sedation tended to be higher in the IVPCA group, as expected given the consumption of more intravenous opioids.

Another limitation of our study was that it did not assess the effect of TAP block on intraoperative consumption of general anesthesia. Preoperative initiation of TAP block could be the subject of future research in patients with hepatic cirrhosis, because TAP block in combination with general anesthesia could provide better intraoperative hemodynamic control, reduce the requirements for both inhalational agents and opioids, and potentially lead to more rapid recovery in this group of patients. A study by Tsuchiya et al came to a similar conclusion in a different group of patients undergoing abdominal surgery but suffering from severe cardiovascular disease. These investigators combined TAP block with general anesthesia and demonstrated better intraoperative hemodynamic stability and early emergence from anesthesia. ${ }^{29}$

Lessons learned from our study are that the use of two catheters to cover both sides and the use of an additional subcostal approach for TAP block, together with a multiorifice catheter to cover a larger area, could improve the efficacy of pain relief and satisfaction scores. Central neuraxial analgesia could provide better satisfaction, but the risks and benefits of each method should be discussed with this population of patients with cirrhosis when scheduled for liver surgery because they have a risk of developing coagulopathy, particularly when liver resection is complicated by major bleeding.

In conclusion, combining TAP block with IVPCA improved postoperative pain management and reduced fentanyl consumption, and this was reflected in a shorter duration of intensive care stay. TAP block can be included as part of a balanced postoperative multimodal pain regimen. Patients should be informed when consented for their postoperative pain management regimen about the risk to benefit ratio of each method. Further multicenter, randomized, controlled studies in larger populations of cirrhotic patients undergoing liver surgery should be designed to investigate the TAP block further before a firm evidence-based conclusion can be reached.

\section{Acknowledgment}

The authors would like to thank all patients involved in this study for their participation, and also our anesthesia residents,
Nagwa Ibrahim, Ghada Mahmoud, and Eman Zaki Awad, for their assistance.

\section{Disclosure}

The authors report no conflicts of interest in this work, which was funded by departmental resources.

\section{References}

1. Itoh $\mathrm{S}$, Uchiyama $\mathrm{H}$, Kawanaka $\mathrm{H}$, et al. Characteristic risk factors in cirrhotic patients for posthepatectomy complications: comparison with noncirrhotic patients. Am Surg. 2014;80(2):166-170.

2. Heilling T. Liver failure following partial hepatectomy. HPB (Oxford). 2006;8:165-174.

3. Niraj G, Kelkar A, Jeyapalan I. Comparison of analgesic efficacy of subcostal transversus abdominis plane blocks with epidural analgesia following upper abdominal surgery. Anaesthesia. 2011;66:465-471.

4. Kadam V, Moran J. Epidural infusions versus transversus abdominis plane (TAP) block infusions: retrospective study. J Anesth. 2011;25: 786-787.

5. El Dawlatly AA, Turkistani A, Kettner SC, et al. Ultrasound guided transversus abdominis plane block: description of a new technique and comparison with conventional systemic analgesia during laparoscopic cholecystectomy. Br J Anaesth. 2009;102:763-767.

6. Jankovic Z. Transversus abdominis plane block: the holy grail of anesthesia for (lower) abdominal surgery. Periodicum Biologorum. 2009;111: 203-208.

7. Tran TMN, Ivanusic JJ, Hebbard P, Barrington MJ. Determination of spread of injectate after ultrasound-guided transversus abdominis plane block: a cadaveric study. Br J Anaesth. 2009;102:123-127.

8. Cross SA. Pathophysiology of Pain. Mayo Clin Proceedings. 1994;69(4):375-383.

9. Ramsay MA, Newman KB, Jacobson RM, et al. Sedation levels during propofol administrations for outpatient colonoscopies. Proc (Bayl Univ Med Cent). 2014;27:12-15.

10. Sharma P, Chand T, Saxena A, Bansal R, Mittal A, Shrivastava U. Evaluation of postoperative analgesic efficacy of transversus abdominis plane block after abdominal surgery: a comparative study. J Nat Sci Biol Med. 2013;4:177-1780.

11. McDonnell JG, O’Donnell B, Curley G, Heffernan A, Power C, Laffey JG. The analgesic efficacy of transversus abdominis plane block after abdominal surgery: a prospective randomized controlled trial. Anesth Analg. 2007;104:193-197.

12. Elkassabany N, Ahmed M, Malkowicz SB, Heitjan DF, Isserman JA, Ochroch EA. Comparison between the analgesic efficacy of transversus abdominis plane (TAP) block and placebo in open retropubic radical prostatectomy: a prospective, randomized, double-blinded study. J Clin Anesth. 2013;25:459-465.

13. Allcock E, Spencer E, Frazer R, Applegate G, Buckenmaier C 3rd. Continuous transversus abdominis plane (TAP) block catheters in a combat surgical environment. Pain Med. 2010;11:1426-1429.

14. Nash H, Khoda B, Heppell S, Turner M. TAP blocks in breast reconstructions using abdominal wall tissue. Anaesthesia. 2011;66:750-751.

15. Sakai T, Manabe W, Kamitani T, Takeyama E, Nakano S. [Ropivacaineinduced late-onset systemic toxicity after transversus abdominis plane block under general anesthesia: successful reversal with $20 \%$ lipid emulsion]. Masui. 2010;59:1502-1505. Japanese.

16. Griffiths JD, Barron FA, Grant S, Bjorksten AR, Hebbard P, Royse CF. Plasma ropivacaine concentrations after ultrasound-guided transversus abdominis plane block. Br J Anaesth. 2010;105:853-856.

17. Neal JM, Bernards CM, Butterworth JF 4th, et al. ASRA Practice Advisory on Local Anesthetic Systemic Toxicity. Reg Anesth Pain Med. 2010;35(2):152-161. 
18. Petersen PL, Mathiesen O, Torup H, Dahl JB. The transversus abdominis plane block: a valuable option for postoperative analgesia? A topical review. Acta Anaesthesiol Scand. 2010;54:529-535.

19. Siddiqui MR, Sajid MS, Uncles DR, Cheek L, Baig MK. A metaanalysis on the clinical effectiveness of transversus abdominis plane block. J Clin Anesth. 2011;23:7-14.

20. Brady RR, Ventham NT, Roberts DM, Graham C, Daniel T. Open transversus abdominis plane block and analgesic requirements in patients following right hemicolectomy. Ann R Coll Surg Engl. 2012;94:327-330.

21. Milan ZB, Duncan B, Rewari V, Kocarev M, Collin R. Subcostal transversus abdominis plane block for postoperative analgesia in liver transplant recipients. Transplant Proc. 2011;43:2687-2690.

22. Sandeman DJ, Bennett M, Dilley AV, Perczuk A, Lim S, Kelly KJ. Ultrasound-guided transversus abdominis plane blocks for laparoscopic appendicectomy in children: a prospective randomized trial. $\mathrm{Br} J$ Anaesth. 2011;106:882-886.

23. Freir NM, Murphy C, Mugawar M, Linnane A, Cunningham AJ. Transversus abdominis plane block for analgesia in renal transplantation: a randomized controlled trial. Anesth Analg. 2012;115:953-957.

24. Zhong T, Wong KW, Cheng H, et al. Transversus abdominis plane (TAP) catheters inserted under direct vision in the donor site following free DIEP and MS-TRAM breast reconstruction: a prospective cohort study of 45 patients. J Plast Reconstr Aesthet Surg. 2013;66:329-336.
25. De Oliveira GS Jr, Fitzgerald PC, Marcus RJ, Ahmad S, McCarthy RJ. A dose-ranging study of the effect of transversus abdominis block on postoperative quality of recovery and analgesia after outpatient laparoscopy. Anesth Analg. 2011;113:1218-1225.

26. Ozalp G, Güner F, Kuru N, Kadiogullari N. Postoperative patientcontrolled epidural analgesia with opioid-bupivacaine mixtures. Can J Anaesth. 1998;45:938-942.

27. Hutchison RW, Chon EH, Gilder R, Moss J, Daniel P. A comparison of a fentanyl, morphine, and hydromorphone patient-controlled intravenous delivery for acute postoperative analgesia: a multicenter study of opioid-induced adverse reactions. Hosp Pharm. 2006;41:659-663.

28. Koo PJ. Postoperative pain management with a patient-controlled transdermal delivery system for fentanyl. Am J Health Syst Pharm. 2005;62:1171-1176.

29. Tsuchiya M, Takahashi R, Furukawa A, Suehiro K, Mizutani K, Nishikawa K. Transversus abdominis plane block in combination with general anesthesia provides better intraoperative hemodynamic control and quicker recovery than general anesthesia alone in high-risk abdominal surgery patients. Minerva Anestesiol. 2012;78:1241-1247.
Local and Regional Anesthesia

\section{Publish your work in this journal}

Local and Regional Anesthesia is an international, peer-reviewed, open access journal publishing on the development, pharmacology, delivery and targeting and clinical use of local and regional anesthetics and analgesics. The journal welcomes submitted papers covering original research, basic science, clinical studies, reviews \& evaluations,

\section{Dovepress}

guidelines, expert opinion and commentary, case reports and extended reports. The manuscript management system is completely online and includes a very quick and fair peer-review system, which is all easy to use. Visit http://www.dovepress.com/testimonials.php to read real quotes from published authors. 